\title{
Psychological care in the function of improving health and quality of life in older age
}

Damir Lučanin, PhD, University of Applied Health Sciences, Zagreb, Croatia; (e-mail: damirl@zvu.hr)

Jasminka Despot Lučanin, PhD, Department of Psychology, Croatian Studies, University of Zagreb, Zagreb, Croatia; (e-mail: jdespot@hrstud.hr)

As people live longer, quality of life becomes an important issue at the individual, family, and social policy levels. Life satisfaction is defined as a person's global assessment of subjective quality of life, or evaluative wellbeing component (Diener, Emmons, Larsen, \& Griffin, 1985; Penezić, 2006; Shin \& Johnson, 1978). Life satisfaction, as an indicator of the person's wellbeing, is one of the indicators of successful psychological adaptation in later life, when a person is usually faced with changes and losses: in health, physical and cognitive functioning, social engagement, and productive activities (Smith \& Ryan, 2016; Schaie, 2016). There is a growing research evidence suggesting that life satisfaction may even be a protective factor in health maintenance, reducing the risk of chronic physical illness and promoting longevity, by way of positive emotions which promote a more active lifestyle and a motivation toward self-care (Carver, Scheier, \& Segerstrom, 2010). Graphic view of the possible role of biological,
psychological and social factors in determining the length
of life and improving the health of the elderly Factors Latent factors Outcomes

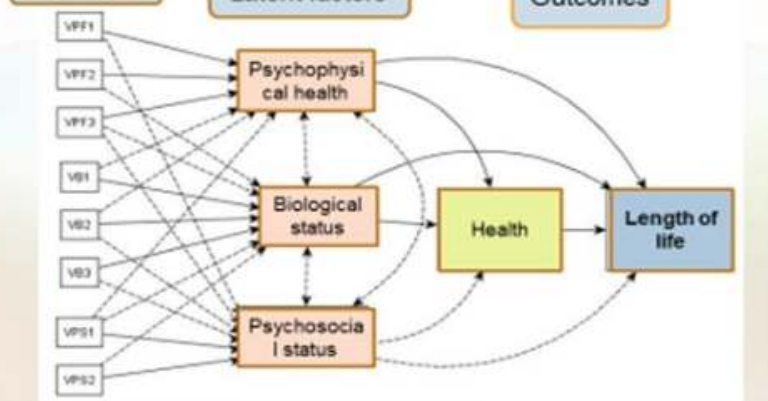

The aim of this paper is to present some findings on the psychosocial factors associated with the health, quality and length of life in old age, that may be applied in the care for the elderly.

We conducted several research on subjective quality of life in older adults in Croatia, on different samples, from which we can recommend several conclusions. In the observed set of psychosocial variables, the strongest predictor of better life satisfaction was better self-perceived health.

\section{Predictors of Life satisfaction in older} adults

The significant predictors of Life satisfaction in older adults, confirmed by other research:

- Self-perceived health,

- Social engagement,

- Depression,

- Functional ability.

(Gutierrez, i ost, 2013; Tomas, Sancho,

Gutierrez i Galiana, 2014; Kim i Sok,

2013)

In our research, the Life satisfaction average scores were:

- Rather high in all participants;

- Not significantly different with regards to the participants' living environment - own home or nursing home.

(Lučanin, Despot Lučanin, Košćec Bjelajac, \& Delale, 2017; Košćec Bjelajac, Despot Lučanin, Lučanin, \& Delale 2019)
With regards to the study design, the significant life satisfaction predictors in older adults were:

* Longitudinal: Depression (neg. corr.) - explained $26 \%$ of variance.

- Cross-sectional: Self-perceived health (pos. corr.) - explained $21 \%$ of variance.

With regards to the older adults' living environment, the significant Life satisfaction predictors were:

* In nursing homes' participants, Selfrated health (pos. corr.) explained $18 \%$ of variance - focus on psychological and health factors

* In participants living at home, Selfrated health and Social participation (pos. corr.) explained $21 \%$ of variance - focus on health and psycho-social factors.
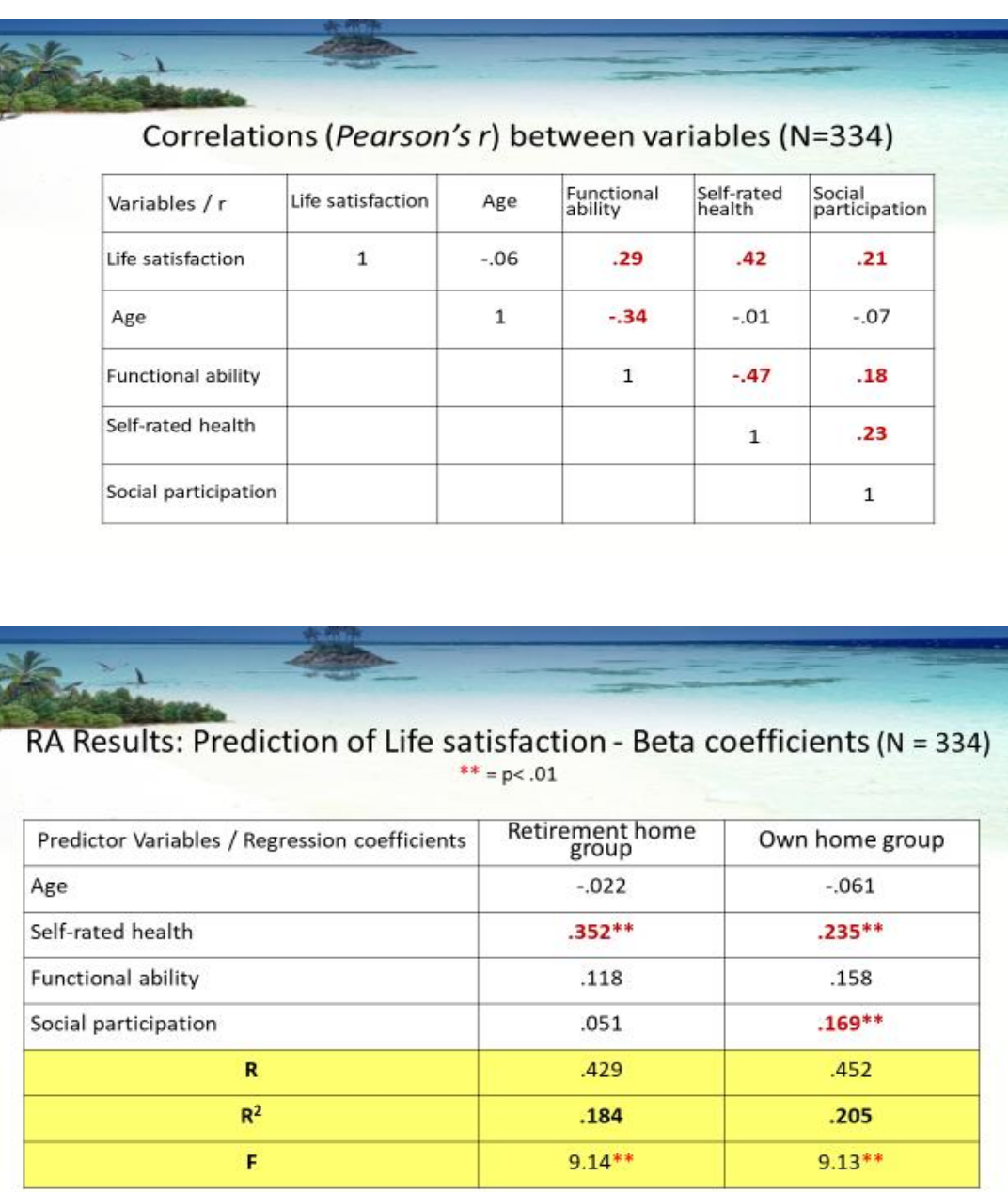

\section{Conclusions}

No differences in life satisfaction were found regarding the participants' living environment, in residential institution or in own home. Also we found that combinations of multivariate analyses confirmed significant contribution of psychosocial variables to the survival prediction in older adults.

The findings support the adaptive processes view of life satisfaction and its determinants, in accordance with the Selection, Optimization and Compensation (SOC) model (Baltes, 1997). The participants enhanced their life satisfaction by focusing on domains of functioning that would ensure higher life-satisfaction in their specific living conditions.

The implications are in the potential improvement of older adults' quality of life, providing psychosocial interventions to enhance the older persons' potential to adapt to wellbeing challenges, adjusted to their living arrangements, and based on their individual functioning and needs.

Implementing both medical and psychological interventions - e.g. depression treatments, cognitive training etc. can have effects on the overall quality of life in old persons. 